\title{
Chemotherapy for Adults with Malignant Glioma: A Systematic Review and Network Meta-Analysis
}

\author{
Wen WANG ${ }^{1}$, Guang $\mathrm{SH}^{2}$, Binbin $\mathrm{MA}^{1}$, Xiangcheng $\mathrm{HAO}^{1}$, Xin $\mathrm{DONG}^{3}$, Bo ZHANG ${ }^{1}$ \\ ${ }^{1}$ The Second Hospital of Dalian Medical University, Department of Neurosurgery, Dalian, China \\ ${ }^{2}$ The Second Hospital of Hebei Medical University, Department of Neurosurgery, Shijiazhuang, China \\ ${ }^{3}$ Tongliao City Hospital, Department of Neurosurgery, Tongliao, China
}

\section{ABSTRACT}

\begin{abstract}
AIM: Malignant glioma is the most common primary brain tumor in adults and the survival rate has remained very low. Thus, determining the optimal treatment for patients can be challenging. To compare the efficacy of common therapies, we performed network meta-analysis to estimate the efficacy and safety among procarbazine, lomustine, vincristine, temozolomide, bevacizumab plus temozolomide, and placebo for patients with malignant glioma.
\end{abstract}

MATERIAL and METHODS: Relevant studies (as of March, 2014) were identified by searching PubMed, Embase, and Central databases. The primary endpoint of the analysis was the overall survival (OS) and progression-free survival (PFS) of glioma patients.

RESULTS: Nine trials with a total of 3472 patients were included in our network meta-analyses. Compared with placebo, bevacizumab plus temozolomide was associated with the highest estimates of OS and PFS for 12 and 24 months (12 month OS odds ratio [OR]: 2.44; 95\% credibility interval [Crls]: 0.76-9.69; 24 month OS OR: 2.56; 95\% Crls: 1.12-5.24; 12 month PFS OR: 6.76; 95\% Crls: 2.80-17.34; 24 month PFS OR: 3.69; 95\% Crls: 0.62-28.63). However, bevacizumab plus temozolomide did not significantly improve OS or PFS compared to temozolomide alone.

CONCLUSION: Bevacizumab plus temozolomide combination therapy is not significantly more effective than temozolomide alone in improving survival of glioma patients. Moreover, bevacizumab was associated with higher hematologic toxicities. Bevacizumab should be used with caution in glioma patients. Additional randomized controlled trials are required to confirm this finding.

KEYWORDS: Bevacizumab, Temozolomide, Chemoradiotherapy, Meta-analysis, Glioma

\section{INTRODUCTION}

$\mathrm{M}$ alignant gliomas are the most common and lethal primary tumors of central nervous system (20), producing serious and progressive disability prior to patient death. Despite the advances in chemoradiotherapy, the survival rate for malignant glioma has remained very low. The majority of glioblastoma patients survive for about two years (17), and only a few patients would survive longer than 5 years (26); WHO III glioma patients would survive ranging from 3 to 5 years (22). Surgery and chemoradiotherapy can improve overall survival (OS) and progression-free survival (PFS), but improvements in long-term survival remain challenging.

Standard therapy for malignant glioma consists of the maximal tumor resection with concomitant chemoradiotherapy. Among chemotherapy agents, temozolomide (TMZ) is the standard agent used in the treatment of malignant glioma (27). TMZ is an oral alkylating agent that has been shown to improve the survival of patients with malignant glioma. However, even with surgery and TMZ therapy, prognosis remains poor and tumor relapse rates remain high. The relatively modest effects 
Wang W. et al: Chemotherapy for Adults with Malignant Glioma

of current standard therapies underscore the urgent need for new therapeutic approaches to treat glioma patients (13).

Recently, it is reported that glioblastomas are highly vascularized because of vascular endothelial growth factor A (VEGFA) (6), which regulates the VEGF signal-transduction pathway (19). Anti-angiogenesis agents have emerged a potential therapeutic strategy for glioma patients. Bevacizumab is a humanized monoclonal anti-body against VEGF and can normalize tumor vasculature (8). Recent evidence supports the use of bevacizumab as a monotherapy for recurrent glioma (16). For example, bevacizumab alone or in combination with chemotherapeutic agents can improve the OS and PFS of glioma patients $(7,11,12)$, particularly when combined with TMZ.

Many therapeutic strategies that for treating malignant gliomas and standard meta-analyses of these approaches have been unable to integrate all randomized controlled trails (RCTs) in one analysis $(1,2,4,7,12,15,21,27,28)$. Furthermore, these analyses include indirect comparisons between different therapeutic strategies. By contrast, network meta-analysis can integrate all RCTs that compare different therapeutic strategies against each other or a placebo group (18). Moreover, network metaanalysis can compare direct and indirect evidence in a network of trials that included multiple interventions and present the relationship of these comparisons (23). This approach integrates all of the relevant evidence to increase the precision of the meta-analysis and produce a more accurate ranking of all of the analyzed therapeutic strategies.

To accurately compare bevacizumab plus TMZ combination therapy (BEV) to other approaches in glioma patients, we performed the network meta-analysis to estimate the efficacy and safety of procarbazine, lomustine, and vincristine (PCV), $\mathrm{TMZ}, \mathrm{BEV}$, and placebo for patients with malignant gliomas.

\section{MATERIAL and METHODS}

\section{Search Strategy}

To identify relevant studies, we searched Pubmed, Embase and the Cochrane databases for systematic reviews published through March 2014 with no language restriction. The Medical Subject Heading and keywords used for the search included: Glioma; Chemotherapy, Adjuvant; Radiotherapy; Procarbazine; Lomustine; Vincristine; Temozolomide; Bevacizumab. We performed a cited references search by using Web of Science and checked the reference lists for both published and unpublished studies. Moreover, we also reviewed the reference lists of published meta-analyses of chemotherapeutic therapies for patients with malignant gliomas. The primary endpoint of this meta-analysis was overall survival (OS) and progression-free survival (PFS). Data extraction was performed by two independent investigators and any discrepancies between selections were resolved through discussion.

\section{Eligibility of Studies}

Inclusion criteria for retrieved studies were: (a) randomized controlled trials of chemoradiotherapy strategies in patients with malignant gliomas (WHO III; IV); (b) randomized treatment allocation; (c) follow-up time of greater than one year; (d) the incidence of events in the study population was specified or could be calculated. Using these criteria, a total of 9 trials were included in our network meta-analyses (Figure 1).

\section{Data Abstraction and Quality Assessment}

Two independent reviewers (WW, BZ) performed data abstraction. We used discussion and consensus to resolve discrepancies. Definitions of the endpoints (OS and PFS) were the same across all trials. The risk of bias in each study was evaluated by using the Cochrane Collaboration's tool. We assessed risk of bias categories based on the items in each study, as follows: sequence generation, allocation concealment, blinding, incomplete data outcomes, selective outcome reporting, and other causes of bias.

\section{Statistical Analysis}

We carried out pair-wise meta-analyses and used the $1^{2}$ statistic to assess the possibility of statistical heterogeneity using the Higgins-Thompson method (14): no heterogeneity: $<25 \%$, low heterogeneity: $25-50 \%$, moderate heterogeneity: $50-75 \%$, high heterogeneity: > 75\%. The Markov Chain Monte Carlo simulation was used to perform multi-treatment metaanalysis within a Bayesian framework (24). All data analyses were performed using Aggregate Data Drug Information System (ADDIS) v1.16.5 (Drugis, Groningen, NL).

We used node-splitting (9) and pair-wise meta-analyses to evaluate the inconsistency of direct comparisons of indirect evidence in the network meta-analysis. The direct and indirect evidence, in accordance in the split node, were analyzed in

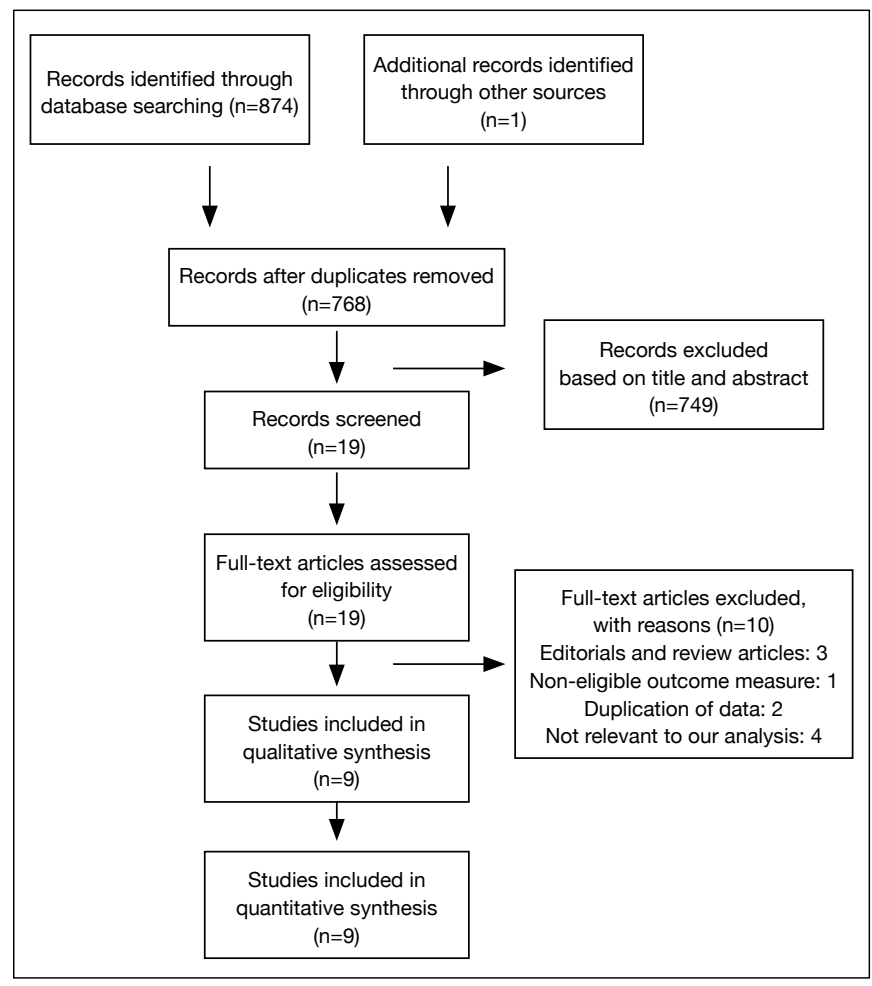

Figure 1: Flow chart indicating the selection process for this meta-analysis. 
a node-splitting assessment. Significant heterogeneity was defined at $p<0.05$. Therapeutic efficacy was assessed based on the odds ratio (OR) with $95 \%$ credibility interval (Crls). Results were considered statistically significant if the Crls did not include 1.0. No statistical adjustment was made for multiple comparisons. The rank probability was calculated and different interventions for each outcome were ranked according to the estimated effect size. The characteristics, relevant outcomes (OS and PFS), mean age of patients, and follow up durations for all trials was recorded.

\section{RESULTS}

\section{Literature Search}

The initial search of all databases for relevant studies yielded 875 publications (301 from PubMed, 261 from Embase, 312 from Central, and 1 additional record from the reference list). These studies were limited to human subjects, RCTs, and published before March 2014. Of these 875 studies, 866 excluded for being duplicate studies or other inconsistencies with the study criteria identified in the title, abstract, of full text of the manuscript. The remaining nine trials were included in the final analysis (Table I).

\section{Study Characteristics}

The nine remaining trials included four different therapeutic strategies tested in malignant glioma patients. Two studies compared bevacizumab plus TMZ combination therapy (BEV) to TMZ therapy alone, one study compared procarbazine, lomustine, and vincristine (PCV) to TMZ therapy. Six of the studies included placebo controls. Two of the placebocontrolled studies assessed the efficacy of PCV on anaplastic oligodendroglioma or anaplastic oligoastrocytoma, while the remaining four compared $\mathrm{TMZ}$ to placebo on glioblastoma. These studies resulted in six theoretical comparisons for each of the primary outcomes. From these studies we established a network of eligible comparisons for the multiple-treatment meta-analysis (Figure 2A) and compared the primary endpoints of OS and PFS (Table II).

\section{Risk of Bias}

We assessed the biases of our included studies using Cochrane Collaboration's tool (Figure 2B, C). Two of the included trials did not describe the method used for generating the allocation sequence $(1,12)$. One trial performed by Oike et al. featured high risk of bias in random sequence generation and allocation concealment but included a historical control (21). In addition, one study did not adequately describe the participants, personnel and outcome assessment, so the risk of bias was considered unclear (27). All the included trials featured low incomplete outcome data, selective reporting, and other biases except for one early closed trial performed by Kocher et al. (15).

\section{Overall Survival}

The primary outcome to assess therapeutic efficacy was OS estimates of glioma patients receiving each therapy. Among the therapies, PCV resulted in the lowest estimate of overall

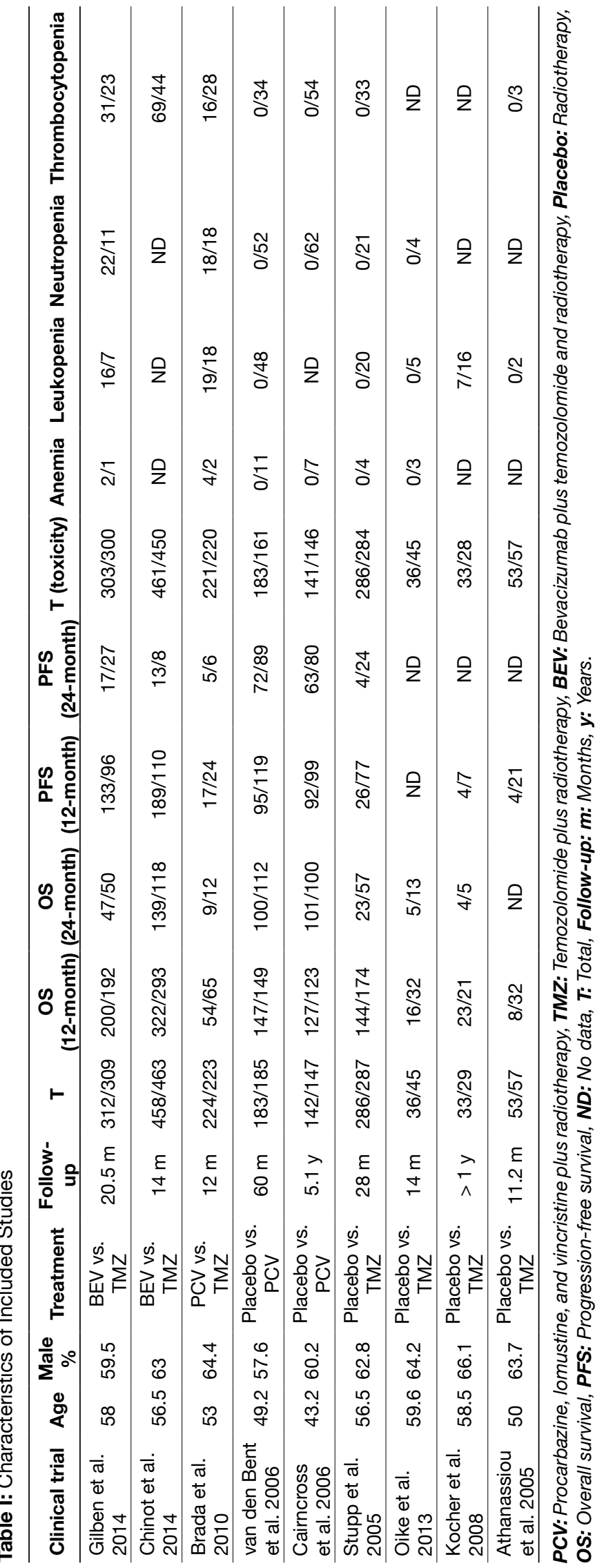




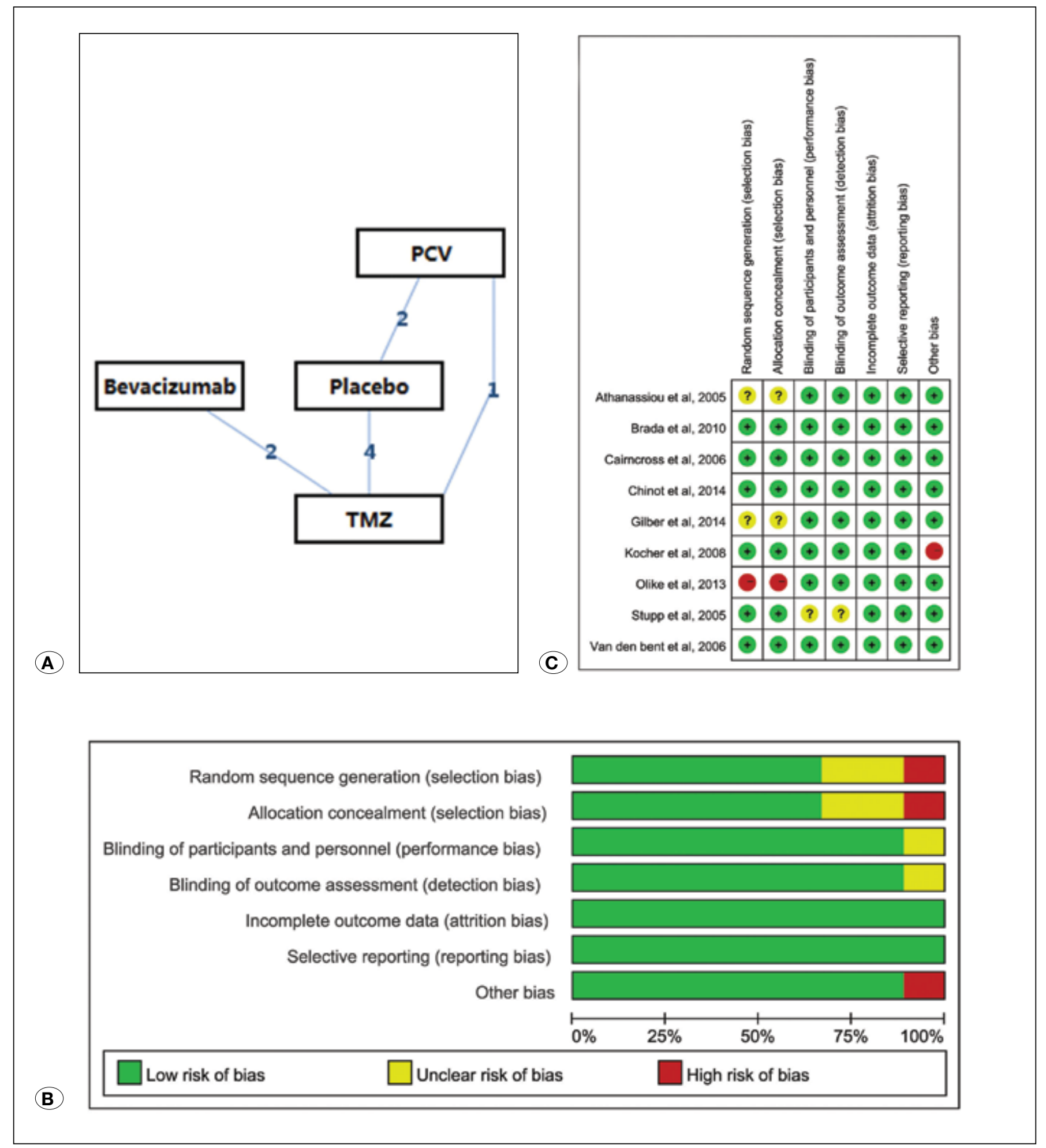

Figure 2: A) Network of eligible comparisons for the meta-analysis. The numbers represent the number of trials comparing each treatment pair. BEV: bevacizumab plus temozolomide and radiotherapy; TMZ: temozolomide plus radiotherapy; PCV: procarbazine, lomustine, and vincristine plus radiotherapy; Placebo: radiotherapy; B) Risk of bias graph; C) Risk of bias summary diagram. 
Table II: Network Meta-Analysis Results

\begin{tabular}{|c|c|c|c|c|}
\hline OS (12-month) & BEV & PCV & Placebo & TMZ \\
\hline BEV & 1 & $0.41(0.09,1.57)$ & $0.41(0.10,1.32)$ & $0.80(0.28,2.26)$ \\
\hline PCV & $2.43(0.64,11.14)$ & 1 & $0.99(0.40,2.44)$ & $1.96(0.80,5.65)$ \\
\hline Placebo & $2.44(0.76,9.69)$ & $1.01(0.41,2.49)$ & 1 & $2.00(1.03,4.63)$ \\
\hline TMZ & $1.25(0.44,3.54)$ & $0.51(0.18,1.25)$ & $0.50(0.22,0.98)$ & 1 \\
\hline OS (24-month) & BEV & PCV & Placebo & TMZ \\
\hline BEV & 1 & $0.45(0.20,1.13)$ & $0.39(0.19,0.89)$ & $0.89(0.52,1.59)$ \\
\hline PCV & $2.20(0.88,4.94)$ & 1 & $0.87(0.50,1.45)$ & $1.96(0.99,3.73)$ \\
\hline Placebo & $2.56(1.12,5.24)$ & $1.14(0.69,1.99)$ & 1 & $2.30(1.27,3.87)$ \\
\hline TMZ & $1.12(0.63,1.92)$ & $0.51(0.27,1.01)$ & $0.44(0.26,0.79)$ & 1 \\
\hline PFS (12-month) & BEV & PCV & Placebo & TMZ \\
\hline BEV & 1 & $0.24(0.09,0.63)$ & $0.15(0.06,0.36)$ & $0.51(0.26,1.00)$ \\
\hline PCV & $4.21(1.59,11.18)$ & 1 & $0.63(0.33,1.16)$ & $2.14(1.06,4.48)$ \\
\hline Placebo & $6.76(2.80,17.34)$ & $1.59(0.86,3.05)$ & 1 & $3.44(1.87,6.53)$ \\
\hline TMZ & $1.96(1.00,3.84)$ & $0.47(0.22,0.94)$ & $0.29(0.15,0.53)$ & 1 \\
\hline PFS (24-month) & BEV & PCV & Placebo & TMZ \\
\hline BEV & 1 & $0.45(0.06,3.00)$ & $0.27(0.03,1.62)$ & $1.08(0.29,3.51)$ \\
\hline PCV & $2.22(0.33,15.62)$ & 1 & $0.61(0.19,1.61)$ & $2.40(0.52,10.41)$ \\
\hline Placebo & $3.69(0.62,28.63)$ & $1.64(0.62,5.25)$ & 1 & $3.99(1.00,18.52)$ \\
\hline TMZ & $0.92(0.29,3.48)$ & $0.42(0.10,1.93)$ & $0.25(0.05,1.00)$ & 1 \\
\hline
\end{tabular}

PCV: Procarbazine, lomustine, and vincristine plus radiotherapy, TMZ: Temozolomide plus radiotherapy, BEV: Bevacizumab plus temozolomide and radiotherapy, Placebo: Radiotherapy, OS: Overall survival, PFS: Progression-free survival.

survival (OR: 1.01; 95\% Crls: 0.41-2.49), followed by TMZ and BEV. However, all therapies extended 12-month OS estimates when compared to placebo, and neither TMZ or BEV was significantly more effective than PCV. A comparison of 24-month OS estimates yielded similar results, with PCV resulting in the lowest OS estimates (OR: 1.14; 95\% Crls: 0.69-1.99). However, BEV and TMZ significantly improved 24-month OS estimates compared to placebo.

\section{Progression-Free Survival}

The analysis of PFS yielded similar results to the analysis of OS estimates for the therapeutic strategies. Among the included therapies, PCV resulted in the lowest 12 and 24-month PFS estimates (12-month OR: 1.59; 95\% Crls: 0.86-3.05; 24-month OR: 1.64; 95\% Crls: 0.62-5.25), followed by TMZ and BEV. However, all the treatments lengthened progressionfree survival compared with placebo. TMZ and BEV were not significantly more effective than PCV in lengthening PFS. Furthermore, there were no significant differences between the PFS estimates of TMZ and BEV.

\section{Hematologic Toxicity}

Analysis of hematologic toxicities among the therapies indicated that PCV, TMZ, and BEV were all more toxic than placebo. Among the therapies, BEV resulted in the highest rate of hematologic toxicity. Furthermore, TMZ resulted in significantly less hematologic toxicity than PCV in glioma patients.

\section{Ranking of Treatments}

Among the therapies, BEV was ranked highest in 12-month OS (70\% chance of being the best therapy), the 24-month OS (70\%), and 12-month PFS (97\%). However, TMZ ranked the highest for 24-month PFS (51\%) (Table III).

\section{Consistency of Network-Model}

No inconsistencies were observed when we compared the effect estimates based on direct versus indirect evidence using node-splitting and pair-wise meta-analysis (Table IV). These data suggest that our model is very robust.

\section{DISCUSSION}

Through this meta-analysis, we assessed efficacy and safety of procarbazine, lomustine, and vincristine (PCV), TMZ, bevacizumab plus $T M Z$ (BEV) and placebo for patients with 
Table III: Ranking Based on Simulations

\begin{tabular}{lllll}
\hline Drug & Rank 1 & Rank 2 & Rank 3 & Rank 4 \\
\hline OS (12-month) & & & & \\
\hline BEV & 0.70 & 0.22 & 0.05 & 0.03 \\
\hline PCV & 0.03 & 0.05 & 0.44 & 0.48 \\
\hline Placebo & 0.01 & 0.03 & 0.47 & 0.49 \\
\hline TMZ & 0.26 & 0.69 & 0.04 & 0.00 \\
\hline OS (24-month) & & & & \\
\hline BEV & 0.70 & 0.26 & 0.02 & 0.01 \\
\hline PCV & 0.02 & 0.03 & 0.70 & 0.26 \\
\hline Placebo & 0 & 0.01 & 0.26 & 0.73 \\
\hline TMZ & 0.28 & 0.70 & 0.02 & 0 \\
\hline PFS (12-month) & & & & \\
\hline BEV & 0.97 & 0.02 & 0 & 0 \\
\hline PCV & 0 & 0.02 & 0.93 & 0.05 \\
\hline Placebo & 0 & 0 & 0.05 & 0.95 \\
\hline TMZ & 0.02 & 0.96 & 0.02 & 0 \\
\hline PFS (24-month) & & & & 0.07 \\
\hline BEV & 0.01 & 0.02 & 0.11 & 0.86 \\
\hline PCV & 0.51 & 0.43 & 0.05 & 0.01 \\
\hline Placebo & 0.44 & 0.10 & 0.04 \\
\hline TMZ & & & 0.74 & 0.08 \\
\hline PCV $P$ & & & & \\
\hline
\end{tabular}

PCV: Procarbazine, lomustine, and vincristine plus radiotherapy, TMZ: Temozolomide plus radiotherapy, BEV: Bevacizumab plus temozolomide and radiotherapy, Placebo: Radiotherapy, OS: Overall survival, PFS: Progression-free survival.

malignant gliomas. Our network meta-analysis indicates that BEV and TMZ all significantly improve 24-month OS and 12-month PFS of glioma patients compared to placebo. However, these therapies were not equal when compared to each other.

When all available treatments were ranked in the network meta-analysis, we observed that BEV was the top ranked therapy with regards to 12 and 24-month OS and 12-month PFS. However, BEV also had the highest rates of hematologic toxicity, even though the primary target of is VEGF Receptor (10). In contrast, TMZ improved 24-month PFS with minimal toxicity. Furthermore, while the differences between OS and PFS with BEV and TMZ therapies were not significantly different. These results suggest that BEV may not be an effective therapy for malignant glioma. Further investigations are warranted to confirm this finding.

Our results are consistent with the current body of evidence supporting the benefits of TMZ for glioma patients. TMZ can improve OS and PFS through various mechanisms. For example, TMZ has a cytotoxic effect on dividing by interfering with DNA replication $(3,5)$. Furthermore, TMZ can sensitize cancer cells to other chemotherapeutic agents (25).

Our analysis indicated that PCV was the least efficient therapy for glioma patients, as 12-month OS and PFS were not significantly improved compared to placebo. Moreover, PCV resulted in significant hematological toxicity. However, PCV did confer a significant survival advantage with regards to 24-month PFS. This result may indicate a delayed benefit for PCV therapy.

Among the trials, two of the RCTs $(4,28)$ assessed the efficacy of PCV on anaplastic oligodendroglioma or anaplastic oligoastrocytoma and one (2) on GBMs and anaplastic oligodendrogliomas grouped together. The prognosis is different for WHO III gliomas, but we focused on the issue that whether bevacizumab could prolong the 12 and 24-month OS and 12 and 24-month PFS on glioblastoma. Moreover, because of the superior model of network meta-analysis, the heterogeneity would decrease (23).

One limitation of our meta-analysis is that it included two RCTs comparing BEV and TMZ but only one trial comparing PCV and $T M Z$. Thus there was less data available to assess the efficacy of PCV. In addition, overestimation or underestimation of the treatment effect is a concern in the smaller trials included in our analysis. Thus, our conclusions regarding the efficacy and safety of these therapeutic strategies should be interpreted with caution. Further, we could not control for the impact of the different treatment details (e.g. drug dosage, radiation time or duration, treatment cycles) in our analysis. In order to more accurately assess the efficacy of these treatments, additional high-quality RCTs will be necessary.

\section{CONCLUSION}

Our meta-analysis indicates that bevacizumab plus temozolomide can improve OS and PFS of malignant glioma patients, but is also associated with high rates of hematologic toxicity. Furthermore, this therapy was not significantly more efficient than temozolomide alone, the standard chemotherapy for glioma patients, which has minimal toxicity. Thus, we suggest that bevacizumab should be used with caution for treating glioma patients.

\section{ACKNOWLEDGEMENTS}

This work was supported in part by National Natural Science Foundation of China (Grant number 81372714). 
Table IV: Node-Splitting and Pair-Wise Meta-Analysis

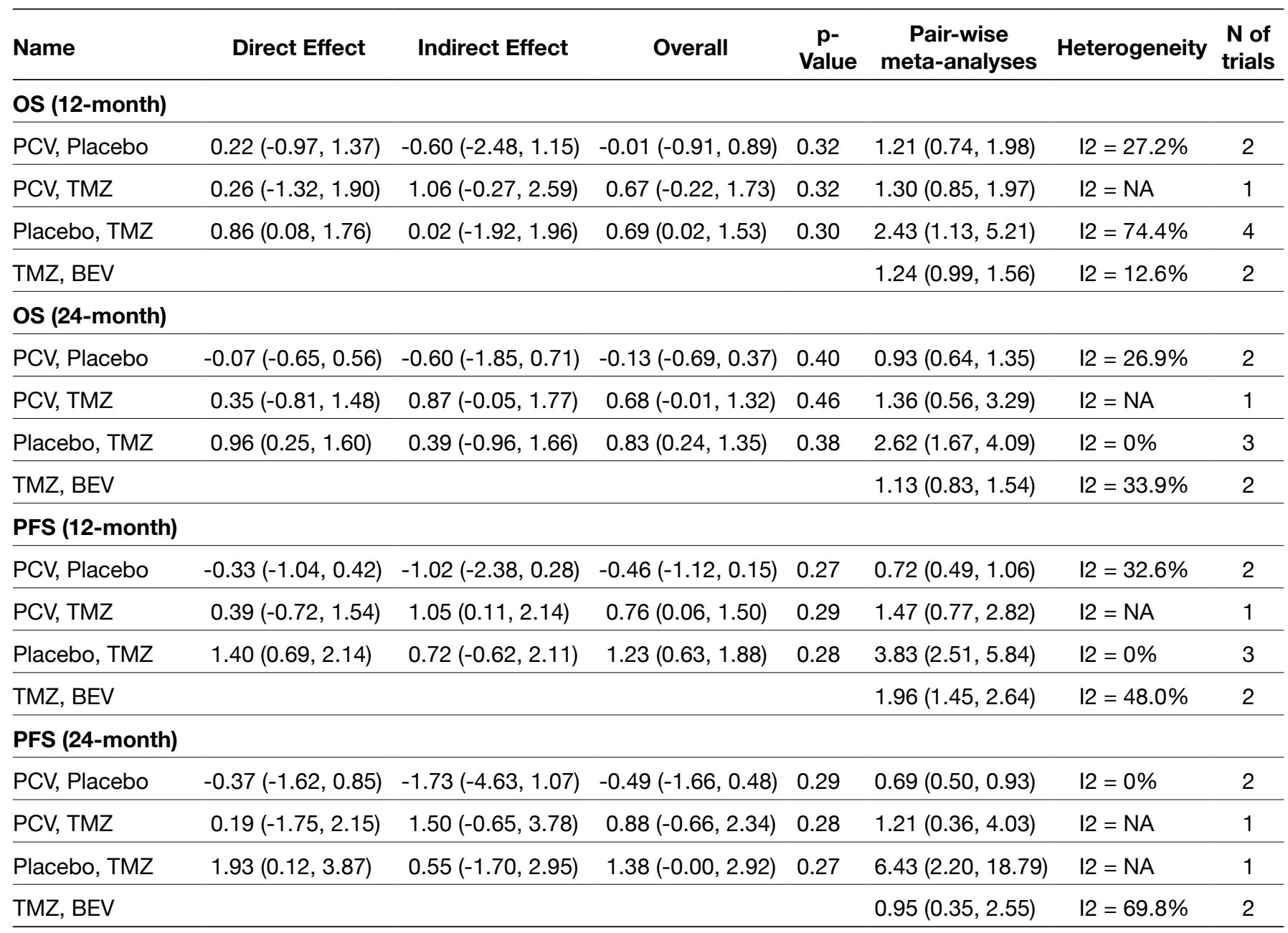

PCV: Procarbazine, lomustine, and vincristine plus radiotherapy, TMZ: Temozolomide plus radiotherapy, BEV: Bevacizumab plus temozolomide and radiotherapy, Placebo: Radiotherapy, OS: Overall survival, PFS: Progression-free survival.

\section{REFERENCES}

1. Athanassiou $H$, Synodinou M, Maragoudakis E, Paraskevaidis M, Verigos C, Misailidou D, Antonadou D, Saris G, Beroukas K, Karageorgis P: Randomized phase II study of temozolomide and radiotherapy compared with radiotherapy alone in newly diagnosed glioblastoma multiforme. J Clin Oncol 23:23722377, 2005

2. Brada M, Stenning S, Gabe R, Thompson LC, Levy D, Rampling R, Erridge S, Saran F, Gattamaneni R, Hopkins K, Beall S, Collins VP, Lee SM: Temozolomide versus procarbazine, lomustine, and vincristine in recurrent high-grade glioma. J Clin Oncol 28: 4601-4608, 2010

3. Brandes AA, Basso U, Reni M, Vastola F, Tosoni A, Cavallo G, Scopece L, Ferreri AJ, Panucci MG, Monfardini S, Ermani M, Gruppo Italiano Cooperativo di Neuro-Oncologia: First-line chemotherapy with cisplatin plus fractionated temozolomide in recurrent glioblastoma multiforme: a phase II study of the Gruppo Italiano Cooperativo di Neuro-Oncologia. J Clin Oncol 22: 1598-1604, 2004
4. Cairncross G, Berkey B, Shaw E, Jenkins R, Scheithauer B, Brachman D, Buckner J, Fink K, Souhami L, Laperierre $\mathrm{N}$, Mehta M, Curran W: Phase III trial of chemotherapy plus radiotherapy compared with radiotherapy alone for pure and mixed anaplastic oligodendroglioma: Intergroup Radiation Therapy Oncology Group Trial 9402. J Clin Oncol 24:27072714, 2006

5. Cavaliere R, Wen PY, Schiff D: Novel therapies for malignant gliomas. Neurol Clin 25:1141-1171, 2007

6. Chi AS, Sorensen AG, Jain RK, Batchelor TT: Angiogenesis as a therapeutic target in malignant gliomas. Oncologist 14: 621-636, 2009

7. Chinot OL, Wick W, Mason W, Henriksson R, Saran F, Nishikawa R, Carpentier AF, Hoang-Xuan K, Kavan P, Cernea D, Brandes AA, Hilton M, Abrey L, Cloughesy T: Bevacizumab plus radiotherapy-temozolomide for newly diagnosed glioblastoma. N Engl J Med 370: 709-722, 2014 
8. de Groot JF, Fuller G, Kumar AJ, Piao Y, Eterovic K, Ji Y, Conrad CA: Tumor invasion after treatment of glioblastoma with bevacizumab: Radiographic and pathologic correlation in humans and mice. Neuro Oncol 12: 233-242, 2010

9. Dias S, Welton NJ, Caldwell DM, Ades AE: Checking consistency in mixed treatment comparison meta-analysis. Stat Med 29: 932-944, 2010

10. Ferrara N, Hillan KJ, Novotny W: Bevacizumab (Avastin), a humanized anti-VEGF monoclonal antibody for cancer therapy. Biochem Biophys Res Commun 333: 328-335, 2005

11. Friedman HS, Prados MD, Wen PY, Mikkelsen T, Schiff D, Abrey LE, Yung WK, Paleologos N, Nicholas MK, Jensen R, Vredenburgh J, Huang J, Zheng M, Cloughesy T: Bevacizumab alone and in combination with irinotecan in recurrent glioblastoma. J Clin Oncol 27:4733-4740, 2009

12. Gilbert MR, Dignam JJ, Armstrong TS, Wefel JS, Blumenthal DT, Vogelbaum MA, Colman H, Chakravarti A, Pugh S, Won $M$, Jeraj R, Brown PD, Jaeckle KA, Schiff D, Stieber VW, Brachman DG, Werner-Wasik M, Tremont-Lukats IW, Sulman EP, Aldape KD, Curran WJ Jr, Mehta MP: A randomized trial of bevacizumab for newly diagnosed glioblastoma. $\mathrm{N}$ Engl $\mathrm{J}$ Med 370: 699-708, 2014

13. Hadziahmetovic M, Shirai K, Chakravarti A: Recent advancements in multimodality treatment of gliomas. Future Oncol 7:1169-1183, 2011

14. Higgins JP, Thompson SG, Deeks JJ, Altman DG: Measuring inconsistency in meta-analyses. BMJ 327: 557-560, 2003

15. Kocher $M$, Frommolt $P$, Borberg $S K$, Rühl U, Steingräber $M$, Niewald M, Staar S, Stuschke M, Becker G, Fischedick AR, Herfarth K, Grauthoff H, Müller RP: Randomized study of postoperative radiotherapy and simultaneous temozolomide without adjuvant chemotherapy for glioblastoma. Strahlenther Onkol 184: 572-579, 2008

16. Kortmann RD: The chemotherapy before or after radiation therapy does not influence survival of children with highrisk medulloblastomas: Results of the multicenter and randomized study of the Pediatric Oncology Group (POG 9031). Strahlenther Onkol 190:106-108, 2014

17. Louis DN, Ohgaki H, Wiestler OD, Cavenee WK, Burger PC, Jouvet A, Scheithauer BW, Kleihues P: The 2007 WHO classification of tumours of the central nervous system. Acta Neuropathol 114: 97-109, 2007

18. Lumley T: Network meta-analysis for indirect treatment comparisons. Stat Med 21:2313-2324, 2002

19. Lund EL, Spang-Thomsen $M$, Skovgaard-Poulsen $H$, Kristjansen PE: Tumor angiogenesis-a new therapeutic target in gliomas. Acta Neurol Scand 97:52-62, 1998

20. Nikiforova MN, Hamilton RL: Molecular diagnostics of gliomas. Arch Pathol Lab Med 135: 558-568, 2011
21. Oike T, Suzuki Y, Sugawara K, Shirai K, Noda SE, Tamaki T, Nagaishi M, Yokoo H, Nakazato Y, Nakano T: Radiotherapy plus concomitant adjuvant temozolomide for glioblastoma: Japanese mono-institutional results. PLoS One 8:e78943, 2013

22. Puduvalli VK, Hashmi M, McAllister LD, Levin VA, Hess KR, Prados M, Jaeckle KA, Yung WK, Buys SS, Bruner JM, Townsend JJ, Davis R, Sawaya R, Kyritsis AP: Anaplastic oligodendrogliomas: Prognostic factors for tumor recurrence and survival. Oncology 65: 259-266, 2003

23. Salanti G, Ades AE, loannidis JP: Graphical methods and numerical summaries for presenting results from multipletreatment meta-analysis: An overview and tutorial. J Clin Epidemiol 64: 163-171, 2011

24. Salanti G, Higgins JP, Ades AE, loannidis JP: Evaluation of networks of randomized trials. Stat Methods Med Res 17: 279-301, 2008

25. Stupp R, Gander M, Leyvraz S, Newlands E: Current and future developments in the use of temozolomide for the treatment of brain tumours. Lancet Oncol 2:552-560, 2001

26. Stupp R, Hegi ME, Mason WP, van den Bent MJ, Taphoorn MJ, Janzer RC, Ludwin SK, Allgeier A, Fisher B, Belanger K, Hau P, Brandes AA, Gijtenbeek J, Marosi C, Vecht CJ, Mokhtari K, Wesseling P, Villa S, Eisenhauer E, Gorlia T, Weller M, Lacombe D, Cairncross JG, Mirimanoff RO; European Organisation for Research and Treatment of Cancer Brain Tumour and Radiation Oncology Groups; National Cancer Institute of Canada Clinical Trials Group: Effects of radiotherapy with concomitant and adjuvant temozolomide versus radiotherapy alone on survival in glioblastoma in a randomised phase III study: 5-year analysis of the EORTC-NCIC trial. Lancet Oncol 10: 459-466, 2009

27. Stupp R, Mason WP, van den Bent MJ, Weller M, Fisher B, Taphoorn MJ, Belanger K, Brandes AA, Marosi C, Bogdahn U, Curschmann J, Janzer RC, Ludwin SK, Gorlia T, Allgeier A, Lacombe D, Cairncross JG, Eisenhauer E, Mirimanoff RO: Radiotherapy plus concomitant and adjuvant temozolomide for glioblastoma. N Engl J Med, 352: 987-996, 2005

28. van den Bent MJ, Carpentier AF, Brandes AA, Sanson $M$, Taphoorn MJ, Bernsen HJ, Frenay M, Tijssen CC, Grisold W, Sipos L, Haaxma-Reiche H, Kros JM, van Kouwenhoven MC, Vecht CJ, Allgeier A, Lacombe D, Gorlia T: Adjuvant procarbazine, lomustine, and vincristine improves progression-free survival but not overall survival in newly diagnosed anaplastic oligodendrogliomas and oligoastrocytomas: A randomized European Organisation for Research and Treatment of Cancer phase III trial. J Clin Oncol 24: 2715-2722, 2006 